\title{
Low-angle Estimation Based on Multi-frequency Maximum Likelihood
}

\author{
Yunhe Cao' ${ }^{1}$, Ting Chen, Shenghua Wang
}

\begin{abstract}
Amony various low-angle estimation methods, MUSIC method and Maximum Likelihood(ML) Method are representative, but the classical MUSIC method can not directly process coherent signal and need spatial smoothing techinique to decorrelate the coherence of the direct and reflected echo, which would reduce spatial resolution ratio. In order to deal with this problem, a new estimation method using multi-frequency signal for low-angle targets is proposed in this paper. First sequential transmission several different frequencies in pulse-to-pulse is applied to decorrelate the coherence of the direct and reflected echo, then the Alternating Projection(AP) Method is combined with Maximum Likelihood Method to simplify the numeration. Finally, the simulation results show that the algorithm can improve the estimation performance of low-angle radar.
\end{abstract}

Keywords: multipath; low-angle estimation; Maximum Likelihood Method; multi-frequency.

\subsection{Introduction}

Low-angle estimation used to estimate the angle of a target flying at a low altitude has attracted a lot of interest for radar researchers. In low-angle estimation, radars receive two or more coherent echoes via multipath (direct path and reflected path)[1], resulting in fades and enhancements of the received data. Generally, the reflected echoes consist of specular and diffuse components [2-6]. If the grazing angle is small and the reflecting surface is smooth, the specular component domi-

\footnotetext{
${ }^{1}$ Yunhe Cao $(\triangle)$

National Laboratory of Radar Signal Processing, Xidian University, China

E-mail: cyh_xidian@163.com
} 
nates, which makes the low-angle problem. In this paper, we will discuss this exceptional case.

The classical maximum likelihood method can distinguish coherent signal. Based on the fitting relationship between the array manifold matrix and the array of receiving data, the angles can be estimated by solving maximum likelihood function. In the case of high signal to noise ratio(SNR), the performance of the method is great, but when the SNR is low, the performance will decline dramatically.

To resolve and exploit multipath effects, the multi-frequency technique is taken into consideration to decorrelate the coherence of the direct and reflected echoes and we propose maximum likelihood based on multi-frequency, which can improve the performance of the classical maximum likelihood method in the case of low SNR. When the SNR is lower than the signal to noise threshold, the performance of the classical maximum likelihood method will decline dramatically. At the same time, when the influence of multipath attenuation is severe, the energy of single frequency signal is very weak and it is not conducive to low-angle estimation. The algorithm provides some robustness to the severe signal fades and makes a proper use of multipath propagation to improve the target detection capability [3]. The algorithm weakens the influence of the multipath attenuation and improves the performance of low-angle estimation.

In addition, the proposed method also can improve the angle distinguishability in multipath environment. The distinguishability of the classical maximum likelihood method will be weakened because of strong coherence of multipath reflected signal, especially when the phase difference between the target echoes and the specular echoes approaches 0 or $\pi$. The proposed method make use of comprehensive information on multi frequency echo, therefore the angle distinguishability in multipath environment is better.

This paper is organized as follows: In Section II, a general mathematics model of spatial spectrum estimation techniques is given. In Section III, the proposed method is described in detail. Simulation results and performance comparison of this method with the classical maximum likelihood method are presented in Section IV. Finally concluding remarks are given.

\subsection{Multipath Model}

To ensure the completeness of the paper, the overall procedure of maximum likelihood method is briefly reviewed. First, let us analyze the low-angle estimation signal model. In maximum likelihood method, we consider a uniform linear array of $\mathrm{M}$ elements in the presence of two echoes from a primary object. So the signal can be expressed as 


$$
\left\{\begin{array}{c}
s_{i}(t)=u_{i}(t) e^{j\left(w_{0} t+\varphi(t)\right)} \\
s_{i}(t-\tau)=u_{i}(t-\tau) e^{j\left(w_{0}(t-\tau)+\varphi(t-\tau)\right)}
\end{array}\right.
$$

Where $u_{i}(t)$ is the amplitude of receives signal, $\varphi(t)$ is the phase of receives signal, $w_{0}$ is the frequency of receives signal. At the same time, we assume the signals are narrow-band so that we can get

$$
\left\{\begin{array}{l}
u_{i}(t-\tau) \approx u_{i}(t) \\
\varphi(t-\tau) \approx \varphi(t)
\end{array}\right.
$$

Combining (1.1) and (1.2), the signal can be expressed as

$$
S_{i}(t-\tau) \approx S_{i}(t) e^{-j \omega_{0} \tau}
$$

Then we can get that the $l t h$ snapshot of the total $\mathrm{N}$ sample of received data is denoted by M-dimensional complex vector, $x_{l}$, which can be expressed as

$$
x_{l}(n)=\sum_{i=1}^{N} g_{l i} s_{i}\left(t-\tau_{l i}\right)+n_{l}(t) \quad l=1,2, \cdots, M
$$

where $g_{l i}$ is the transmission gain between the $l$ th snapshot of the array and the $i t h$ singal, $n_{l}(t)$ is noise, $\tau_{l i}$ is the time difference between the snapshot of the array and reference snapshot.

In this case, the array of M elements can be expressed asollows

$$
\left[\begin{array}{c}
x_{1}(t) \\
x_{2}(t) \\
\vdots \\
x_{M}(t)
\end{array}\right]=\left[\begin{array}{cccc}
g_{11} e^{-j w_{0} \tau_{11}} & g_{12} e^{-j w_{0} \tau_{12}} & \cdots & g_{1 N} e^{-j w_{0} \tau_{1 N}} \\
g_{21} e^{-j w_{0} \tau_{21}} & g_{22} e^{-j w_{0} \tau^{22}} & \cdots & g_{2 N} e^{-j w_{0} \tau_{2 N}} \\
\vdots & \vdots & & \vdots \\
g_{M 1} e^{-j w_{0} \tau_{M 1}} & g_{M 2} e^{-j w_{0} \tau_{M 2}} & \cdots & g_{M N} e^{-j w_{0} \tau_{M N}}
\end{array}\right]\left[\begin{array}{c}
s_{1}(t) \\
s_{2}(t) \\
\vdots \\
s_{N}(t)
\end{array}\right]+\left[\begin{array}{c}
n_{1}(t) \\
n_{2}(t) \\
\vdots \\
n_{M}(t)
\end{array}\right]
$$

In ideal condition, the channel of every element in the array is the same and $g_{l i}$ can be ignored. So (1.4) can be simplified as

$$
\left[\begin{array}{c}
x_{1}(t) \\
x_{2}(t) \\
\vdots \\
x_{M}(t)
\end{array}\right]=\left[\begin{array}{cccc}
e^{-j w_{0} \tau_{11}} & e^{-j w_{0} \tau_{12}} & \cdots & e^{-j w_{0} \tau_{1} N} \\
e^{-j w_{0} \tau_{21}} & e^{-j w_{0} \tau^{2}} & \cdots & e^{-j w_{0} \tau_{2 N} N} \\
\vdots & \vdots & & \vdots \\
e^{-j w_{0} \tau_{M 1}} & e^{-j w_{0} \tau_{M 2}} & \cdots & e^{-j w_{0} \tau_{M N}}
\end{array}\right]\left[\begin{array}{c}
s_{1}(t) \\
s_{2}(t) \\
\vdots \\
s_{N}(t)
\end{array}\right]+\left[\begin{array}{c}
n_{1}(t) \\
n_{2}(t) \\
\vdots \\
n_{M}(t)
\end{array}\right]
$$

And (1.6) can equivalent to the expression as follows 


$$
X(t)=A S(t)+N(t)
$$

Where $X(t)$ and $N(t)$ are an $M \times 1$ matrix, $S(t)$ is a $N \times 1$ matrix, and the ith column vector of the $M \times N$ matrix $A \square\left[a\left(\theta_{1}\right), a\left(\theta_{2}\right), \cdots, a\left(\theta_{N}\right)\right]$ can be expressed as

$$
a\left(\theta_{i}\right)=\left[\begin{array}{c}
\exp \left(-j \omega_{0} \tau_{1 i}\right) \\
\exp \left(-j \omega_{0} \tau_{2 i}\right) \\
\vdots \\
\exp \left(-j \omega_{0} \tau_{M i}\right)
\end{array}\right]=\left[\begin{array}{c}
\exp \left(\frac{-j 2 \pi \cdot z_{1} \sin \theta_{i}}{\lambda}\right) \\
\exp \left(\frac{-j 2 \pi \cdot z_{2} \sin \theta_{i}}{\lambda}\right) \\
\vdots \\
\exp \left(\frac{-j 2 \pi \cdot z_{M} \sin \theta_{i}}{\lambda}\right)
\end{array}\right] \quad i=1,2, \cdots, N
$$

Where $\lambda$ is the wavelength, $z_{k}$ is the position of the $m t h$ element in the array。

\subsection{Proposed Method}

In this section, we propose an estimation method based on Maximum Likelihood Method and each step of the proposed method is described in detail. As the preconfition, several assumptions are made in the algorithm as following: (1) the noise is temporally white and circularly symmetric zero-mean complex Gaussian random variable;(2) the noise in different cells is mutually statistically independent; (3) all the multipath delayed signals from the target lie within the range cell under consideration; (4) the sample data in varied frequency pulses is mutually statistically independent since the frequency bandwidth is wide enough.

\subsubsection{Classical Maximum Likelihood Method}

Based on those assumptions, we can get

$$
\begin{gathered}
E\left\{x\left(t_{i}\right)\right\}=A(\theta) s\left(t_{i}\right) \\
E\left\{\left(x\left(t_{i}\right)-\bar{x}\left(t_{i}\right)\right)\left(x\left(t_{j}\right)-\bar{x}\left(t_{j}\right)\right)^{H}\right\}=\sigma^{2} I \delta_{i j} \\
E\left\{\left(x\left(t_{i}\right)-\bar{x}\left(t_{i}\right)\right)\left(x\left(t_{j}\right)-\bar{x}\left(t_{j}\right)\right)^{T}\right\}=0
\end{gathered}
$$

The joint PDF can be expressed as: 


$$
f(\bar{X})=\prod_{i=1}^{L} \frac{1}{\pi \operatorname{det}\left[\sigma^{2} \vec{I}\right]} \cdot \exp \left(-\frac{1}{\sigma^{2}}\left|\vec{x}\left(t_{i}\right)-\vec{A}(\vec{\theta}) \vec{s}\left(t_{i}\right)\right|^{2}\right)
$$

So we can get the $f(\bar{X})$ logarithm as follow

$$
-\operatorname{Inf}(\vec{X})=L \cdot \operatorname{In} \pi+M L \cdot \operatorname{In} \sigma^{2}+\frac{1}{\sigma^{2}} \sum_{i=1}^{L}\left|x_{i}-\vec{A}(\theta) \vec{s}\left(t_{i}\right)\right|^{2}
$$

The equality (1.11) is an function about the unknown parameters $\theta, \sigma^{2}$ and $s$, so we can get the maximum likelihood estimation as follow

$$
\begin{gathered}
\widehat{\sigma}^{2}=\frac{1}{M} \operatorname{tr}\left\{P_{A}^{\perp} \hat{R}\right\} \\
\widehat{s}=A^{+} \vec{x}
\end{gathered}
$$

Where $P_{A}$ is the projection matrix of $A(\theta), \hat{R}$ is the estimate of covariance matrix.

Finally, replacing $\sigma^{2}$ and $S$ in (1.11) with (1.12), we obtain

$$
\widehat{\theta}=\max _{\theta, s_{i}}\left\{\operatorname{tr}\left\{P_{A}^{\perp} \widehat{R}\right\}\right\}=\max _{\theta, s_{i}}\left\{\operatorname{tr}\left\{P_{A} \widehat{R}\right\}\right\}
$$

The Angle of target is obtained by calculating the equality (1.13).

\subsubsection{Alternating Projection(AP) Method}

Alternating Projection (AP) method is an alternating optimization method based on the projection matrix decomposition. Alternating optimization method is a kind of resolutions to simple multi-dimensional optimization problems and this method is achieved by iteration. When every step of the iteration is done, a parameter is optimized and the other parameters remain the same. So the $\hat{\theta}_{i}^{(k+1)}$ can be obtained by the one dimensional optimization problem:

$$
\widehat{\theta}_{i}^{(k+1)}=\max _{\theta_{i}} \operatorname{tr}\left(P_{\left[A\left(\widehat{\Theta}_{(i)}^{(k)}\right), a\left(\widehat{\theta}_{i}\right)\right]} \widehat{R}\right)
$$

Where $\mathrm{P}$ is the alternating projection matrix, $\mathrm{R}$ is the covariance matrix, $\Theta_{i}^{(k)}$ is the calculated parameter whose dimension is $N-1$ and can be expressed as:

$$
\Theta_{(i)}^{(k)}=\left[\widehat{\theta}_{1}^{(k)}, \cdots, \widehat{\theta}_{i-1}^{(k)}, \widehat{\theta}_{i+1}^{(k)}, \cdots, \widehat{\theta}_{N}^{(k)}\right]
$$


In the optimization of each parameter, this algorithm can find the extreme of its likelihood function at axial orientation and the speed depends on the characteristics of the likelihood function near the peak. A kind of effective initialization method is introduced simply as follow:

First of all, the first signal DOA estimation can be expressed as:

$$
\widehat{\theta}_{1}^{(0)}=\max _{\theta_{1}} \operatorname{tr}\left(P_{a\left(\hat{\theta}_{1}\right)} \widehat{R}\right)
$$

Then, assume that the first signal is located in $\widehat{\theta}_{1}^{(0)}$, the second signal DOA estimation can be expressed as:

$$
\widehat{\theta}_{2}^{(0)}=\max _{\theta_{2}} \operatorname{tr}\left(P_{\left[a\left(\hat{\theta}_{1}^{0}\right), a\left(\hat{\theta}_{2}\right)\right]} \widehat{R}\right)
$$

According to the above method, when the ith iteration is done, the other calculated parameters remain the same. Then we can get $\widehat{\theta}_{i}^{(0)}$ until $\widehat{\theta}_{N}^{(0)}$, and then using the equality (1.14) until the iterative frequency or the search accuracy is attained. Alternating Projection (AP) method can greatly reduce the optimization calculation by simplifying an extremely complex multidimensional search problem to some simple one-dimensional search problems.

\subsubsection{Maximum Likelihood Based on Multi-frequency echoes}

When using multiple frequency emission signal, we can obtain the maximum likelihood estimate by the follow equality:

$$
\widehat{\theta}_{i}^{(k+1)}=\max _{\theta_{i}} \operatorname{tr}\left(\sum_{q=1}^{Q} P_{\left[A\left(\widehat{\Theta}_{(i)}^{(k)}\right), a\left(\widehat{\theta}_{i}\right)\right]}^{q} \widehat{R}^{q}\right)
$$

Where $Q$ is the number of the carrier frequency. Maximum likelihood based on multi-frequency not only can remove the peak which is caused by the single frequency, but also can be equivalent to improve SNR. Here notice that the large frequency spacing is needed to decorrelate the coherence of the direct and reflected echo.

\subsection{Performance Comparison}

In this section, we give two part simulations to validate the proposed maximum likelihood based on multi-frequency and we compare the performance of the proposed method to the conventional method by numerical simulations. The first part simulation compares the proposed method with the classical maximum likelihood 
to illustrate its advantages. The second part simulation compares the proposed method with multiple frequency monopulse estimation to analyse the scope of application.

In the following simulations, the number of carrier frequencies $N$ is 5 . In order to meet the fairness of two algorithms comparation, we consider two algorithms have the same time, that means SNR under the condition of single frequency is higher $10 \lg 5$ than that under the condition of the proposed method and $10 \lg 5$ is about $7 \mathrm{~dB}$. The simulation parameter is set as follow: number of array elements: $\mathrm{N}=110$; interval of array elements: $\mathrm{d}=\mathrm{lambda} / 2$; relation between the surface distance and the target height: $\mathrm{R}=600 \mathrm{~km}-700 \mathrm{~km}, \mathrm{ht}=30 \mathrm{~km} ; \mathrm{R}=700 \mathrm{~km}$ $800 \mathrm{~km}$, ht $=35 \mathrm{~km}$; number of snap: snap=1; repeat frequency: 50 times; when under the condition of single frequency, SNR: $7 \mathrm{~dB}$ 、 frequency: $5.5 \mathrm{GHZ}$; when under the condition of multifrequency, SNR:0dB 、 frequency: $5.2-5.8 \mathrm{GHZ}$.

We can know from Figs. 1 that classical ML method needs multi-dimensional search, its error estimation of the angle at the beginning is as good as multifrequency ML. But with the increase of the distance, the angle is smaller and smaller, the result of the proposed method has better estimation precision. And its distinguish performance becomes poorer in far away places due to the fact that disparity in angle between the direct signal and the reflected signal is small. In addition, multi-frequency ML can fade away the peak and make the MSE of every point smaller, which should be equivalent to the effect of higher SNR.

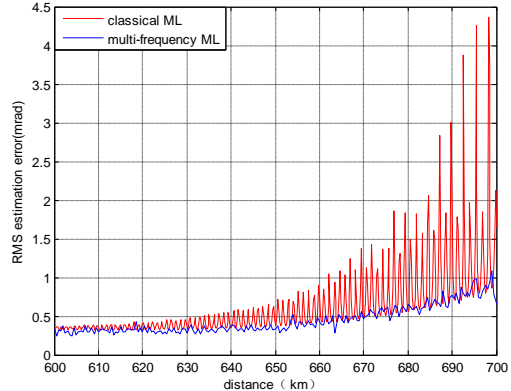

(a) $\mathrm{ht}=35 \mathrm{~km}$

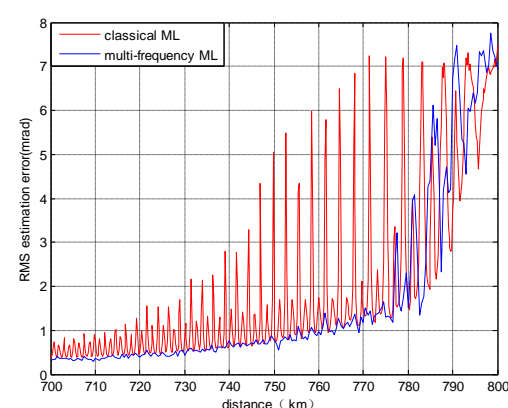

(b) $\mathrm{ht}=35 \mathrm{~km}$

Fig. 1.1 root mean square(RMS) estimation error

To further demonstrate the performance of the proposed method, we compare it with multiple frequency monopulse estimation method. It can be found from Figs. 2 that multiple frequency monopulse estimation has many peaks, which can cause serious error. However, the simulation curve of multi-frequency ML is relatively smooth. But with the increase of the distance, estimation precision of the proposed method in Fig.2(b) (when the range larger than $770 \mathrm{~km}$ ) becomes worse. The reason is that disparity in angle between the direct signal and the reflected signal is small enough and beyond this array ability. 


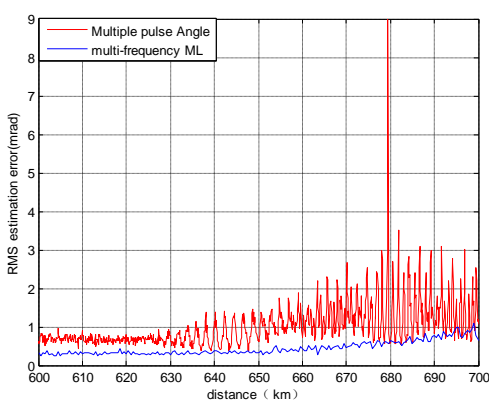

(a) $\mathrm{ht}=35 \mathrm{~km}$

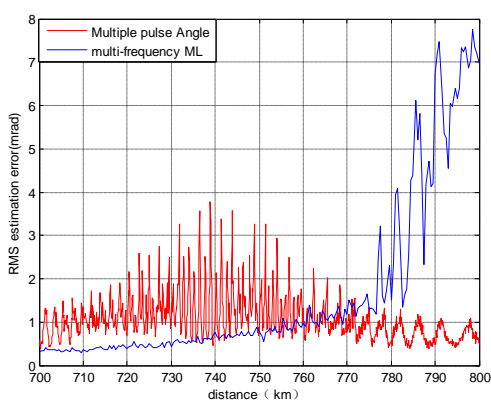

(b) $\mathrm{ht}=35 \mathrm{~km}$

Fig. 1.2 RMS estimation error

\subsection{Conclusion}

This paper proposed a new low-angle estimation method based on the multifrequency technique to improve low-angle estimation performance when existing multipath reflections. The proposed method has better estimation precision and less computational load by adopting Alternating Projection (AP) method.

\section{Acknowledgments}

This work was supported in part by the National Natural Science Foundation of China $(61372136,61172137$, and 61271290) and in part by the Fundamental Research Funds for the Central Universities (K5051202005, and K5051302089).

\subsection{References}

1. Sebt,M.A.,Sheikhi,A.,Nayebi,M.M., Robust low-angle estimation by an array radar,IET Radar Sonar Navig.,2010,4,(6),pp.780-790.

2. Barton,D.K., Low angle radar tracking, Proc.IEEE, 1974,62,(6), pp. 687-740

3. K. B. Yu, Recursive super-resolution algorithm for low-elevation target angle tracking in multipath, Proc. IEE Radar, Sonar Navigation, 1994, 141(4), pp. 223-228.

4. Harun Taha Hayvaci, Antonio De Maio, Danilo Erricolo, Improved detection probability of a radar target in the presence of multipath with prior knowledge of the environment[J], IET Radar Sonar Navig., 2013, Vol. 7, Iss. 1, pp. 36-46.

5. Z. Tarique, W. Q. Malik, D. J. Edwards, Bandwidth requirements for accurate detection of direct path in multipath environment, Electronics Letters, 19th January 2006.42.

6. Jincan Ding, Haowen Chen, Baoyi You, Fluctuating target detection in low-grazing angle with Multi-input Multiple-output radar[J]. ICSP 2012 Proceedings, pp. 1714-1719. 